\title{
Recurrent rheumaticactivity in rheumatic heart disease
}

\author{
Sarkar M K ${ }^{1}$, Mishra $\mathbf{R}^{2}$, Pandya $\mathbf{P}^{3}$, Chellani A A ${ }^{4}$, Ghosh Nelson ${ }^{5}$ \\ ${ }^{1}$ Dr. Manuj Kumar Sarkar, Assistant Professor, Department of Medicine, Index Medical College Hospital and Research \\ Centre, Indore, MP, India, ${ }^{2}$ Dr. Rishu Mishra, ${ }^{3}$ Dr. ParthPandya, ${ }^{4}$ Dr. Ankit Anil Chellani, ${ }^{5}$ Dr. Nelson Ghosh. ${ }^{(2-}$ \\ ${ }^{5)}$ Postgraduate Resident, Department of Medicine, Index Medical College Hospital and Research Centre, Indore, MP, \\ India.
}

Address for Correspondence: Dr. Manuj Kumar Sarkar, Assistant Professor, Department of Medicine, Index Medical College Hospital and Research Centre, Indore, MP, India, Email: manojsarkar654321@gmail.com

\begin{abstract}
Introduction:Acute rheumatic fever (ARF) is caused by autoimmune effect of group A streptococcal infection (GAS). Cardiac involvement is typically pancarditis. Valvular damage persists after the improvement of acute episode of ARF, the persistent valvular defect and its sequel is called rheumatic heart disease (RHD). People with previous episode of ARF are at increased risk of developing newer episodes of ARF and it increases the severity of valvular damage. Thus RHD gradually worsens in patients with recurrent attacks of ARF. Frequent episodes of ARF can be prevented by secondary prophylaxis.Methods and Materials:All diagnosed cases of RHD were includedand non RHD patients were excluded from this study. Proper history, detailed physical examination, routine laboratory test, ECG and echocardiography were done in all patients. Recurrent rheumatic activity was diagnosed on the basis of modified Jones criteria'1992 and who criteria of 2004.Results:Among 100 patients studied, 45 cases were hospitalised because of heart failure due to poor compliance of medications, 38 had acute attack of rheumatic fever, and 17 cases were having lower respiratory tract infection. Thus the incidence of acute rheumatic activity in established RHD was 38\%. 50\% patients were on penicillin prophylaxis and $10 \%$ were on oral tablets of penicillin or azithromycin for secondary prophylaxis. It means $60 \%$ of the patient population was on secondary prophylaxis. Conclusion: Recurrent rheumatic activity is a hidden cause of morbidity and mortality in established cases of RHD as penicillin prophylaxis is not adequate because of poor compliance to the drug.
\end{abstract}

Keywords:Acute rheumatic fever, Rheumatic Heart Disease, Recurrent Rheumatic Activity, penicillin prophylaxis.

\section{Introduction}

Acute rheumatic fever (ARF) is caused by autoimmune effect of group A streptococcal infection (GAS). Joint mainly large joints, cardiac tissues, skin and brain can be involved in ARF.Any type of cardiac tissues can be involved in ARF, means it is typically pancarditis. Involvement of endocardium causes valvulitis, involvement of myocardium leads to myocarditis, which can ultimately cause myocardial failure, involvement of pericardium leads to pericarditis and pericardial effusion. However, valvular damage persists even after the improvement of acute episode of ARF, the persistent valvular defect and the sequel of ARF is called rheumatic heart disease (RHD). People with previous episode of ARF are at increase risk of developing newer episodes of acute rheumatic fever and

Manuscript received: $12^{\text {th }}$ Feb 2016 Reviewed: $23^{\text {rd }}$ Feb 2016

Author Corrected: $04^{\text {th }}$ March 2016

Accepted for Publication: 14 $4^{\text {th }}$ March 2016 this kind of recurrent episodes of ARF can increase the severity of damaged valves. Thus RHD gradually worsens in these patients who develop multiple attacks of ARF [1]. A recent study on group A streptococcal infection suggested that globally 15.6 million patients are having RHD, 470000 new cases are being detected each year, and 230000 dies due to RHD each year [2]. Rheumatic heart disease develops in $30-45 \%$ of patients who had suffered from acute rheumatic fever [3]. As $\mathrm{ARF}$ and RHD both are high prevalent in developing countries, the most common cardiac disease in childhood is RHD in the developing world.The Jones criteria is divided into major and minor manifestations along with supporting evidence of precedingstreptococcal infection [4], still it was difficult to diagnose recurrent rheumatic activity in established 
heart disease. In 2001, WHO provide guidelines for diagnosing first and recurrent episodes of rheumatic fever in such patients using Jones criteria [1]. Recurrent ARF leads to gradually progressive form of RHD. Detection of acute rheumatic fever early and administering secondary prophylaxis with antibiotics is very important aspect of prevention of RHD [5].

The incidence of acute rheumatic activity in diagnosed cases of RHD is the most important factor in increasing the severity of valvular damage and thus deteriorating the patient's status. Secondary prophylaxis for prevention of recurrent rheumatic fever is also paramount important factor for RHD prognosis in our country. Our study will show the incidence of recurrent rheumatic activity in RHD and it will also find out the compliance of patients to secondary prophylaxis in preventing recurrent rheumatic activity.

\section{Methods and Materials55}

The study was done in Index Medical College Hospital and Research Center for a period of one year from January 2015 to December 2015. The Institute is a tertiary care center in Indore, Madhya Pradesh, India and it provides best available treatment in all fields by qualified doctors of various subjects. A total of 100 patients were studied. All patients with already diagnosed with rheumatic heart disease who visited outpatient department(OPD) and who were admitted in our hospital specially in ICCU and wards for their problems related to their cardiac illness were included for the study. Patients unrelated to cardiac symptoms or signs that were not thought to be due to recurrent rheumatic fever or RHD were excluded from the study. Proper history and detailed physical examinations were done in all patients. Females were always examined in presence of a female nurse or a female attendant after taking consent. Routine laboratory test were done in all patients, particularly to look for leukocytosis, raised ESR, and raised ASO titer. ECG was done in all patients to look for prolonged PR interval. Echocardiography was performed by expert cardiologist for detailed evaluation of cardiac status. Special attention was given to valve structure and function, presence of any regurgitation or stenosis, any new appearance of lesion compared with previous echocardiography report, presence of pericardial effusion and presence of any signs of heart failure. If any one of the above abnormalities were present, it was considered to be due to recurrent rheumatic activity unless there were other causes for these changes were found. Recurrent rheumatic activity was diagnosed on the basis of modified Jones criteria'1992 and who criteria of 2004.

\section{Result}

The following results were observed from this study. $60 \%$ patients were female, $40 \%$ were male. Most common age group of RHD was 20-30 years, a total of 44 patients were in this group, followed by equal frequency in 10-20 and 30-40 years of age group, which was $25 \%$ each and $10 \%$ cases were more than 40 years of age. Reason for hospitalization was worsening heart failure, lower respiratory tract infection, and recurrent attacks of rheumatic fever. 45 cases had heart failure mainly due to stoppage of medications, 38 had acute attack of rheumatic fever, and 17 cases were having lower respiratory tract infection. Based on modified Jones criteria a total of 38 cases of recurrent rheumatic fever cases were diagnosed. Thus the incidence of acute rheumatic activity in established RHD was $38 \%$.

$50 \%$ patients were on IM injection of benzathine penicillin and $10 \%$ patients were taking oral tablets of penicillin or azithromycin for secondary prophylaxis. It means $60 \%$ of the patients population was covered for prevention of acute rheumatic fever and rests $40 \%$ were unprotected. History confirmed that the compliance to intramuscular injection of penicillin was the main reason for not taking secondary prophylaxis. 32 cases of recurrent rheumatic fever was found in those patients who were not taking prophylaxis, it means, $80 \%$ cases of ARF were in unprotected group, it suggest that penicillin prophylaxis is very important in prevention of recurrent attacks of rheumatic fever. Newer valvular lesions were found in 25 patients, most commonly severe MR, mild to moderate AR, pericardial effusion was found in 8 patients, unexplained congestive heart failure was present in 7 patients. ASO titer was raised in all the 38patients with recurrent rheumatic fever. All the patients with recurrent rheumatic fever had preceding history of streptococcal infection in the form of sore throat.

\section{Discussion}

Acute rheumatic fever is a disease of pediatric age group, most commonly it is found in 5-15 years of age, but the incidence of acute rheumatic fever has come down dramatically because of improvement of hygienic conditions and extensive use of broad spectrum antibiotic. Still RHD is frequently encountered in our country. Rheumatic heart disease (RHD) develops after 
cardiac involvement in acute rheumatic fever, is a very common cause of cardiovascular mortality and morbidity [6, 7, and 8]. $35 \%$ to $40 \%$ of hospital admissions for cardiac causes are because of RHD, and it is a very important indication for cardiac surgery in developing countries [6]. In $80 \%$ of patients, ARF resolves within 12 weeks [9].

Patients with past history of acute rheumatic fever need long-term follow-up and the myocardium becomes weak and susceptible for reinfection throughout life, but it depends on the severity of carditis in previous episode. But within 5 years of primary episode, most recurrences occurs [10]. Severity of carditis in acute rheumatic fever is directly proportional the severity of chronic valvular disease in RHD and severecarditis is associated with more frequent recurrence [11]. This recurrent rheumatic fever can be prevented by adequate implementation of secondary prophylaxis with either intramuscular penicillin injection or oral penicillin tablet or macrolide group of antibiotics. World Health Organization (WHO) recommended that intramuscular long acting penicillin (benzathinepenicillin) should be given for secondary prophylaxis unless it is contraindicated or compliance is poor, in these patients oral tablets should be used [12]. If patients are not willing to take intramuscular injection, then oral penicillin tablet twice daily can be given as an alternative, however intramuscular injection is better than oral tablet in reducing recurrent rheumatic fever [13]. Prophylaxis with oral penicillin tablet is associated with poorer treatment adherence and it may lead to inappropriate serum concentration [14].

\section{Conclusion}

This study confirmed the importance of penicillin prophylaxis in prevention of recurrent attacks of acute rheumatic fever in established RHD. The incidence of ARF has decreased in recent time because of extensive use of antibiotics and improvement in hyegenic status, even in our country, it is still a hidden cause of morbidity and mortality in established cases of RHD as penicillin prophylaxis coverage is not adequate because of poor compliance to the drug. From our study it can be concluded that despite the incidence of acute rheumatic fever has come down in our country, but RHD is still prevalent in India because of high incidence of recurrent rheumatic fever in these established RHD cases and the high incidence of recurrent rheumatic fever is because of poor compliance to penicillin. The recurrent rheumatic activity can be prevented by secondary prophylaxis.

Funding: Nil

Conflict of interest: Nil

Permission from Institutional Research Board (IRB): Yes

\section{References}

1. WHO Expert Consultation on Rheumatic Fever and Rheumatic Heart Disease. Rheumatic fever and rheumatic heart disease: report of a WHO Expert Consultation, Geneva, 29 Oct - 1 Nov 2001. Geneva: WHO, 2004. (WHO Technical Report Series No. 923.)

2. Carapetis JR, Steer AC, Mulholland EK, Weber M.The global burden of group A streptococcal diseases.Lancet Infect Dis. 2005 Nov; 5(11):685-94.

3. Guilherme L, Cury P, Demarchi LM, Coelho V, Abel L, Lopez AP, Oshiro SE, Aliotti S, Cunha-Neto E, Pomerantzeff PM, Tanaka AC, Kalil J.Rheumatic heart disease: proinflammatory cytokines play a role in the progression and maintenance of valvularlesions.Am J Pathol. 2004 Nov; 165(5):1583-91.

4. Guidelines for the diagnosis of rheumatic fever. Jones criteria, 1992 update. Special Writing Group of the Committee on Rheumatic Fever Endocarditis and Kawasaki Disease of the Council on Cardiovascular Disease in the Young of the American Heart Association.JAMA 1992 Oct 21; 268(15): 2069-73.

5. Smith MT, Zurynski Y, Lester-Smith D, Elliott E, Carapetis J.Rheumatic fever - identification, management and secondary prevention.AustFam Physician. 2012 Jan-Feb; 41(1-2):31-5.

6. Rheumatic fever and rheumatic heart disease. Report of a WHO Study Group.World Health Organ Tech Rep Ser. 1988; 764:1-58.

7. Vijaykumar M, Narula J, Reddy KS, Kaplan EL.Incidence of rheumatic fever and prevalence of rheumatic heart disease in India.Int J Cardiol. 1994 Mar 1; 43(3):221-8.

8. Padmavati S.Rheumatic fever and rheumatic heart disease in developing countries.Bull World Health Organ. 1978; 56(4):543-50. 
9. Diagnosis and management of acute rheumatic fever; Chapter 3, The Australian guideline for prevention, diagnosis and management of acute rheumatic fever and rheumatic heart disease (2nd edition), 2012.

\section{Carvalho SM, Dalben I, Corrente} JE, Magalhães CS.Rheumatic fever presentation and outcome: a case-series report.Rev Bras Reumatol. 2012 Mar-Apr; 52(2):241-6.

11. Meira ZM, Goulart EM, Colosimo EA, Mota CC.Long term follow up of rheumatic fever and predictors of severe rheumatic valvar disease $i$ n Brazilian childrenand adolescents.Heart. 2005 Aug;9 1(8):1019-22.
12. Beggs $\mathrm{S}$ et al; Antibiotic use for the Prevention and Treatment of Rheumatic Fever and Rheumatic Heart Disease in Children, WHO, 2008 June 30.

13. Manyemba J, Mayosi

BM.Penicillin for secondary prevention of rheumatic fever.Cochrane Database Syst Rev. 2002 ; 3):CD002227. Penicillin for secondary prevention of rheumatic fever.

14. Dajani AS.Adherence to physicians' instructions as a factor in managing streptococcal pharyngitis.Pediatrics. 1996 Jun; 97(6Pt 2):976-80.

\section{How to cite this article?}

Sarkar M K, Mishra R, Pandya P, Chellani A A, Ghosh Nelson Recurrent rheumaticactivity in rheumatic heart diseas. Int J Med Res Rev 2016;4(3):353-356. doi: 10.17511/ijmrr.2016.i03.11 